\title{
Velocity determination of hydrogen clusters at a cluster-jet target
}

\section{Alexander Täschner*, Alfons Khoukaz, Esperanza Köhler, and Hans-Werner Ortjohann}

Institut für Kernphysik, Westfälische Wilhelms-Universität Münster, Germany

E-mail: taschna@uni-muenster.de

The prototype of the cluster-jet target station for PANDA has been built up at the University of Münster. This setup allows for systematic studies on the production of high density cluster-jet beams and their properties. One important parameter determining the performance of internal targets for storage ring experiments is the target thickness. In case of the cluster-jet targets the target thickness is closely related to the mean velocity of the clusters. In this contribution we will present a technique developed for the determination of the velocity distribution of the clusters. Results obtained with this method will be shown and compared to calculations based on different gas dynamic models for the gas flow through the nozzle of the cluster source. Furthermore we will present first results of an extension of this technique which allows for the determination of the mass distribution of the clusters.

Supported by EU (FP6 and FP7), BMBF (06MS253I and 06MS9149I), and GSI F+E.

8th International Conference on Nuclear Physics at Storage Rings-Stori11,

October 9-14, 2011

Laboratori Nazionali di Frascati dell'INFN, Italy

\footnotetext{
* Speaker.
} 


\section{Introduction}

Cluster-jet targets are important internal targets in storage ring experiments. For future $4 \pi$ experiments, e.g. the PANDA experiment at the future accelerator centrum FAIR in Darmstadt in Germany, a cluster-jet target with a target thickness of above $10^{15}$ atoms $/ \mathrm{cm}^{2}$ have been developed at the University of Münster [1, 2, 3]. In these targets the clusters are produced from cold compressed hydrogen gas with temperatures around $25 \mathrm{~K}$ and pressures of about $18 \mathrm{bar}$. This purified hydrogen is pressed through a de Laval nozzle with a minimum diameter of $\approx 28 \mu \mathrm{m}$. Through the expansion of the gas into a first vacuum chamber clusters are produced from the fluid. In order to separate the gas from the cluster-jet two skimmers are used. These skimmers separate different pumping stages and thus form a differential pumping system. The second skimmer is called collimator and is additionally used to define the shape and size of the cluster-jet at the interaction point with the beam in the storage ring.

One of the most important properties of internal targets is the target thickness. In case of the cluster-jet target the density can be measured by movable rods mounted in the scattering chamber which are shown in Fig. 1. The rod diameter of $d=1 \mathrm{~mm}$ is small with respect to the size of about $10 \mathrm{~mm}$ of the cluster-jet. Clusters colliding with these rods are stopped and increase the vacuum pressure in this chamber by evaporation. In Fig. 2 a typical measurement is presented, where the vacuum pressure increase is plotted as function of the rod position. Assuming a specific volume density distribution $\rho(x, y)$ this pressure profile can be described by the following equation [1]:

$$
p(x)=p_{\mathrm{b}}+\frac{v R T}{S M} \int_{x-x_{0}-d / 2}^{x-x_{0}+d / 2} \mathrm{~d} x^{\prime} \int_{-\infty}^{+\infty} \mathrm{d} y^{\prime} \rho\left(x^{\prime}, y^{\prime}\right) .
$$

In this equation $p_{\mathrm{b}}$ is the background pressure, $R$ the universal gas constant, $M$ the molar mass of the gas, $S$ the pumping speed of the used pumping system, and $v$ the mean velocity of the clusters.

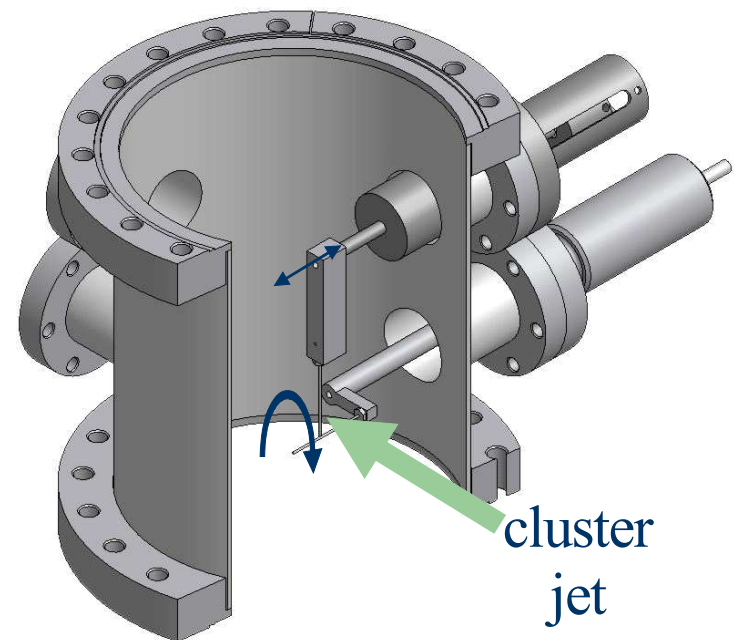

Figure 1: Section view of the scattering chamber with the movable rods used to measure the clusterjet profile.

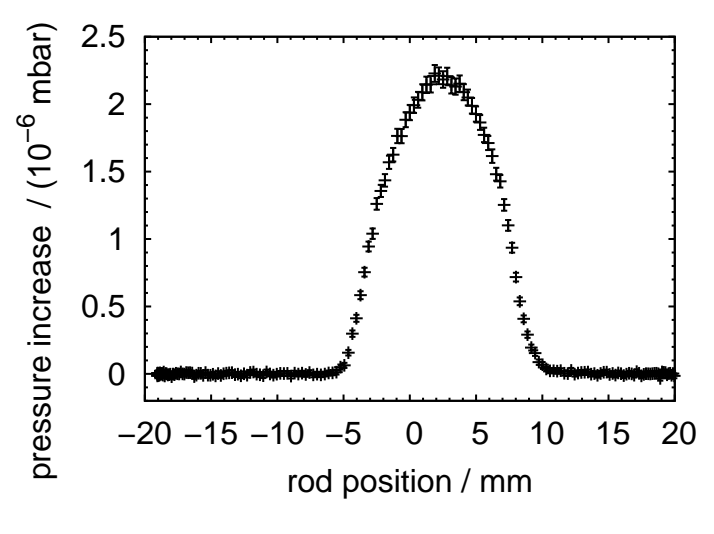

Figure 2: Example of the pressure increase in the scattering chamber as function of the position of the movable rod. 


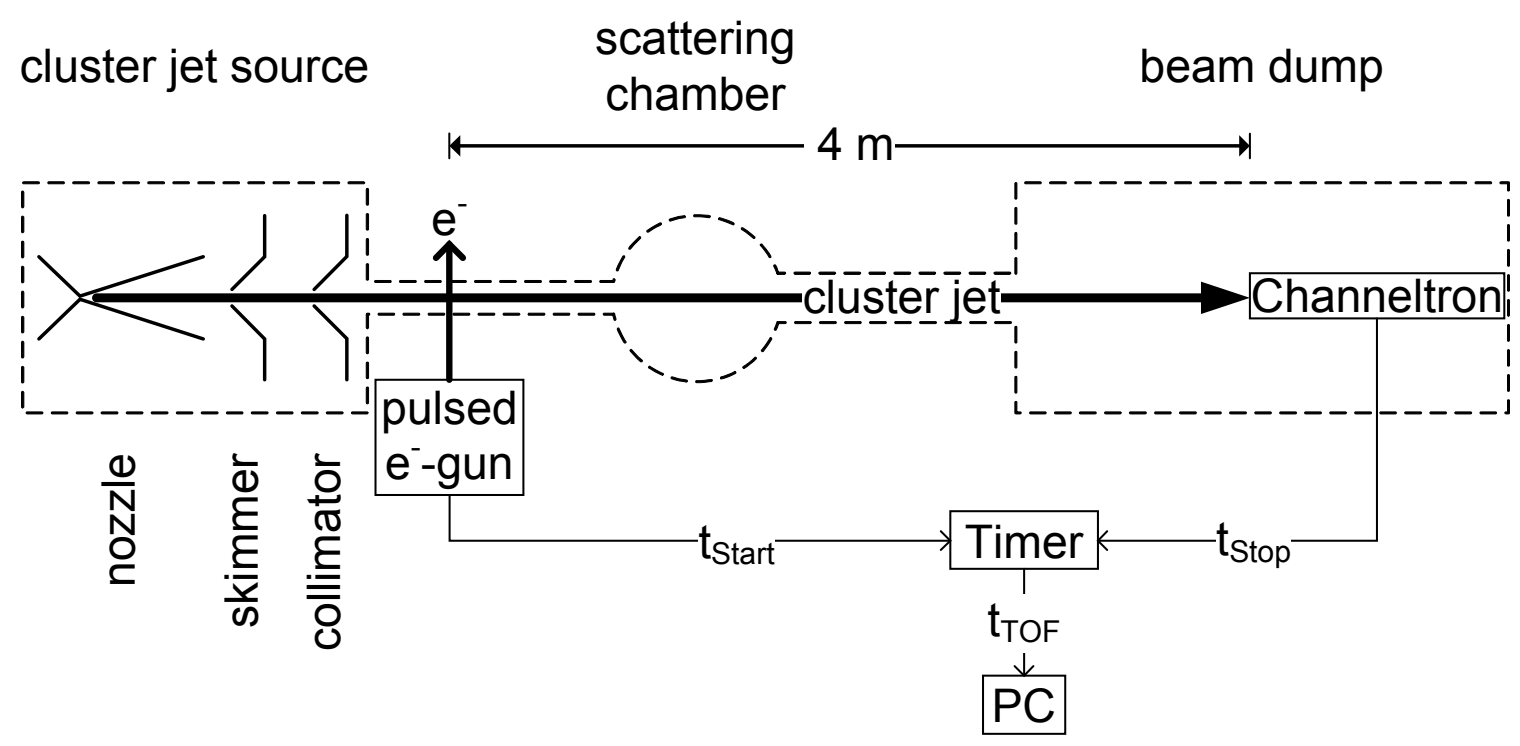

Figure 3: Schematic view of the time-of-flight setup for the velocity determination of single clusters.

Using this equation the target thickness can be calculated by adjusting the free parameters of the assumed density distribution to the measured pressure profiles.

At conventional cluster-jet targets, e.g. the former target for the E835 experiment at FERMI$\mathrm{LAB}$, the mean velocity of the clusters were measured and it was found, that the velocities can be described with the maximum velocity $v_{\max }$ of perfect gas accelerated in an isentropic expansion through a convergent-divergent nozzle [4]:

$$
v_{\max }=\sqrt{\frac{2 \kappa}{\kappa-1} \frac{R T_{0}}{M}}
$$

where $T_{0}$ is the gas temperature at the inlet of the nozzle. These measurements were done at a pressure below 8 bar and temperatures between $15 \mathrm{~K}$ and $40 \mathrm{~K}$ where the hydrogen is in the gas phase. Since the pressure and temperature at the Münster target operated with maximum target thickness, corresponds to the liquid phase of hydrogen, it is very important to measure the velocity distribution at these operation conditions and to compare them to the gas phase conditions. For this reason a dedicated system was designed and installed to measure the velocity distribution of the clusters at the Münster cluster-jet target.

\section{Time-of-flight setup}

In Fig. 3 the schematic view of the constructed time-of-flight setup is shown. Single clusters produced in the cluster source are ionized by a pulsed electron gun mounted at a short distance behind the collimator. This electron gun is pulsed with a repetition rate of $\approx 20 \mathrm{~Hz}$ and a pulse duration of $\approx 20 \mu \mathrm{s}$. The electron current is adjusted in a way that for each pulse at most one cluster is ionized. The ionized clusters are detected by a Channeltron after a flight path of around $4 \mathrm{~m}$. The start and stop pulses are detected by a timer system based on a MC9S08QG8 microcontroller by Freescale Semiconductor and the time difference is send to a computer. 


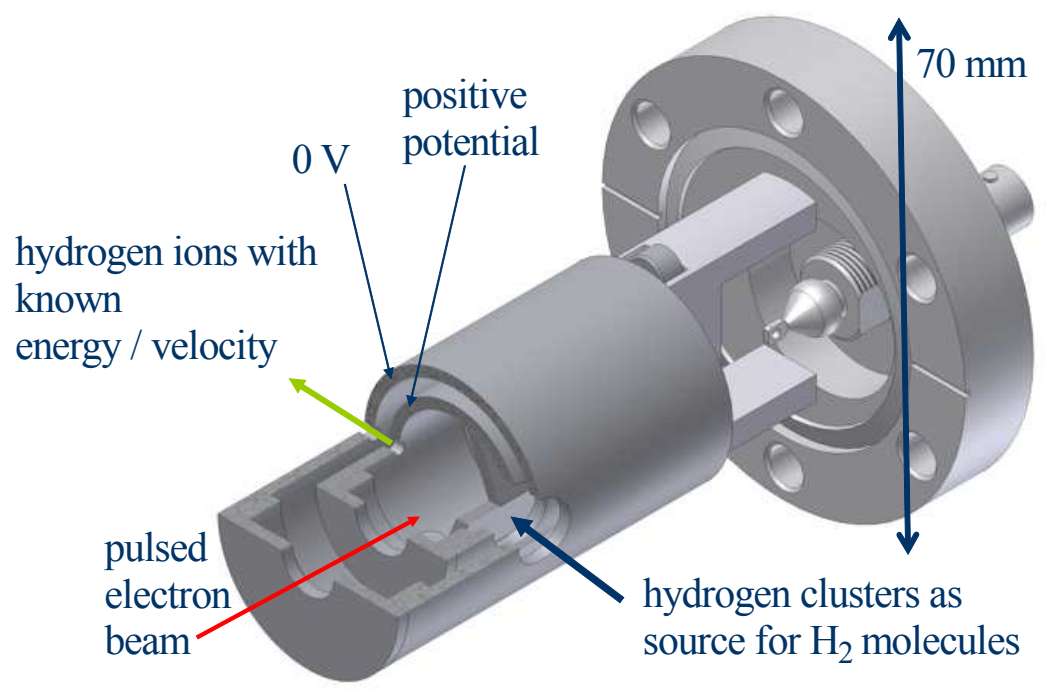

Figure 4: Three-quarter view of the calibration source used to produce hydrogen ions with known kinetic energy.

Since the time delays introduced by the pulser and the detection electronics are unknown the time-of-flight setup has to be calibrated. For this a calibration source shown in Fig. 4 was used. The source consist of two nested cylinders. The outer cylinder is electrically grounded and the inner cylinder is connected to a voltage source of up to $4 \mathrm{kV}$. The calibration source is mounted in such a way, that the cylinder axis is perpendicular to the axis of the cluster beam, so that the clusters can enter it through a hole with a diameter of $10 \mathrm{~mm}$. In the inner cylinder the clusters are stopped by a wedge shaped plate producing hydrogen gas. This gas is ionized by the pulsed electron beam entering along the axis of the two cylinders. The potential difference between the cylinders accelerate ions to known kinetic energy through a $2 \mathrm{~mm}$ hole along the cluster beam axis.

In Fig. 5 an example of a time-of-flight distribution is shown which was measured using the calibration source with an acceleration voltage of $100 \mathrm{~V}$. In this distribution four peaks from different ion species are clearly visible. The peak with the lowest mean time-of-flight can be attributed to photons produced in the cluster source. Since their time-of-flight is around $10 \mathrm{~ns}$ the measured time of around $4 \mu \mathrm{s}$ is a direct consequence of the time offset caused by the electronics. The three other peaks correspond to different hydrogen ions, namely $\mathrm{H}^{+}, \mathrm{H}_{2}^{+}$, and $\mathrm{H}_{3}^{+}$. The time offset and the length of the flight path between electron gun and Channeltron could be extracted by measuring the mean time-of-flight for the different ions as function of the acceleration voltage. For these measurements the electron gun was operated with a repetition rate of about $25 \mathrm{kHz}$ and a pulse duration of $\approx 2 \mu \mathrm{s}$. With this calibration setup a time resolution of about $3 \mu$ s was reached which is predominantly given by the pulse duration of the electron gun.

\section{Comparison of experimental data with simulation}

Using the presented time-of-flight method the velocity distribution of hydrogen clusters produced in the Münster cluster-jet target were measured. Three examples for the measured time-offlight distributions of hydrogen clusters are presented in Fig. 6. The distributions were measured 


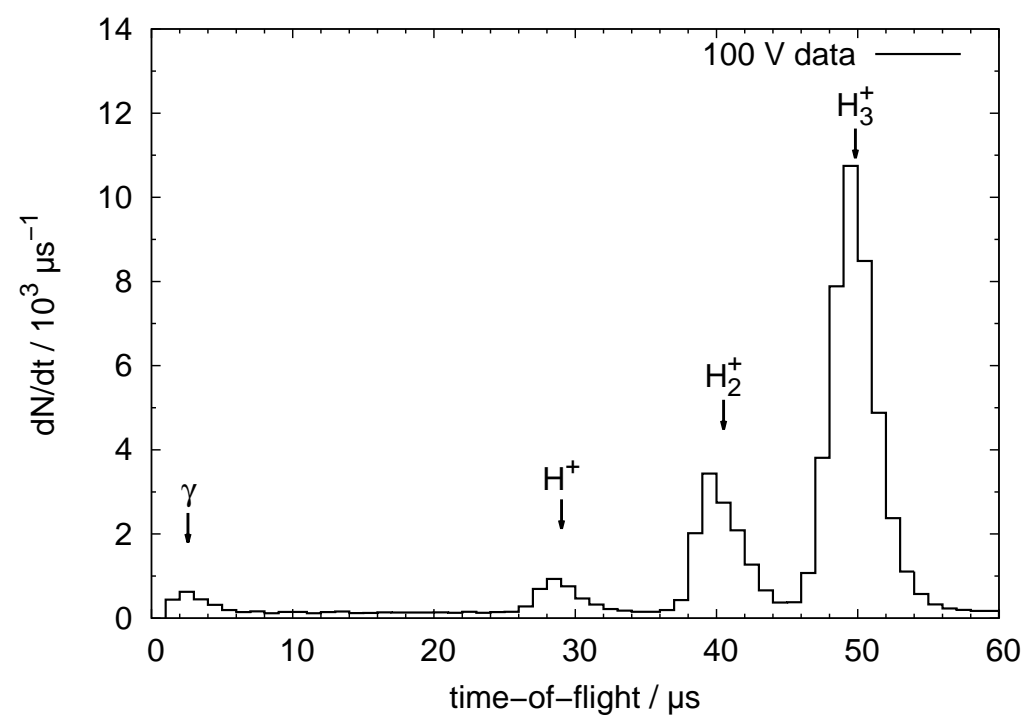

Figure 5: Example of a time-of-flight distribution using the calibration source.

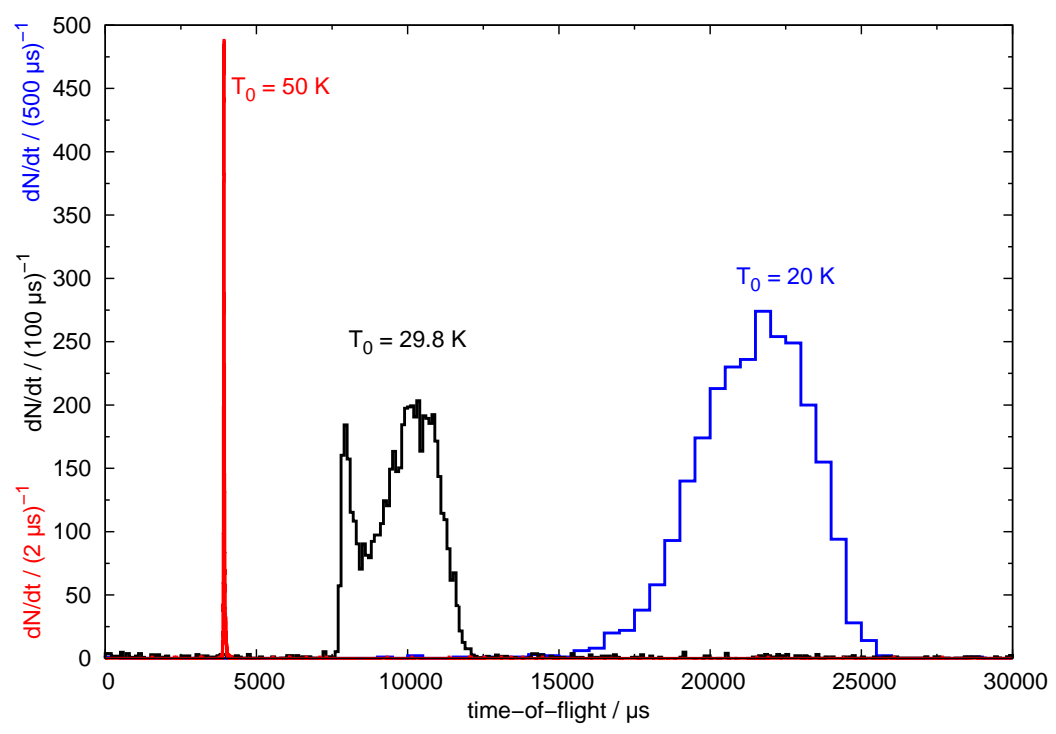

Figure 6: Time-of-flight distribution of hydrogen clusters produced in the Münster cluster jet target at three different hydrogen temperatures at the nozzle inlet. All distributions were measured with the same pressure of 8 bar at the inlet.

at a constant hydrogen pressure of 8 bar at the nozzle inlet, but at different temperatures of $20 \mathrm{~K}$, $29.8 \mathrm{~K}$ and $50 \mathrm{~K}$. The distribution at highest temperature is very narrow with a width $(1 \sigma)$ of around $19 \mu$ s which increases up to $1800 \mu$ s at $20 \mathrm{~K}$. Near the boiling temperature of $30 \mathrm{~K}$ a double peak structure was observed with a small peak at higher velocity on top of a broad peak with a lower mean velocity indicating the production from two coexisting phases. In Fig. 7 the mean cluster velocity is plotted as function of the hydrogen temperature at the inlet of the nozzle at a constant hydrogen pressure of 8 bar. The bold solid line indicates the maximum velocity of the perfect gas 


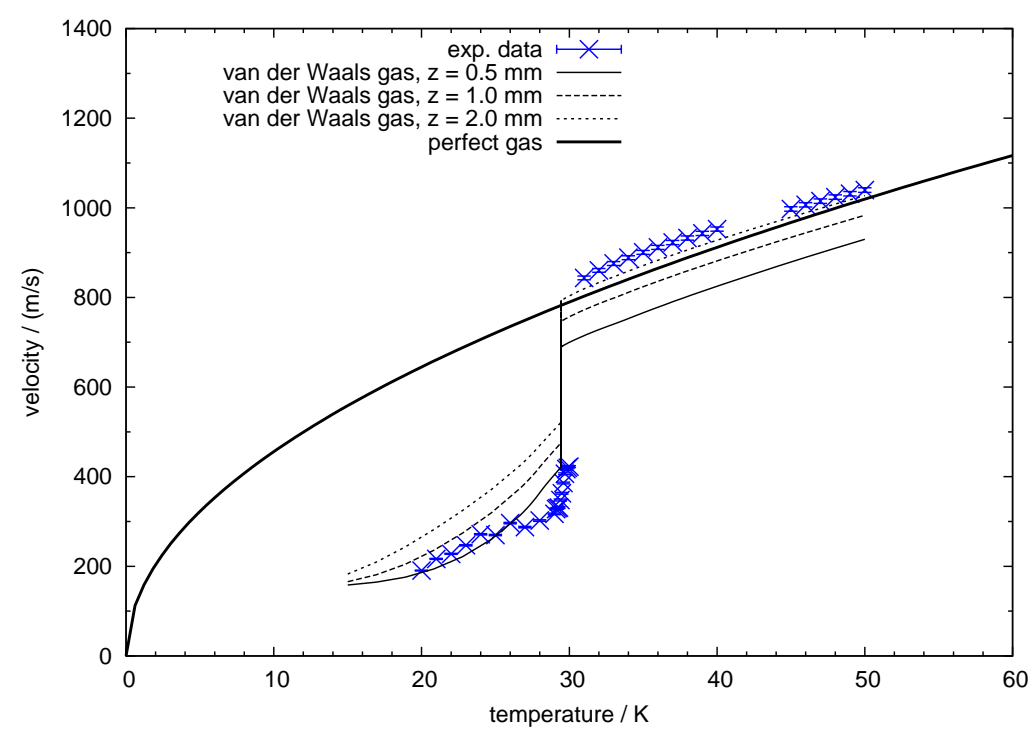

Figure 7: Mean velocity of hydrogen clusters produced in the Münster cluster jet target as function of the hydrogen temperature at the nozzle inlet. All data points were taken with the same pressure of $8 \mathrm{bar}$ at the inlet. The bold solid line corresponds to model calculations based on the perfect gas model. The fine solid, dashed and dotted line are calculated using the van der Waals equation of state at different positions behind the nozzle throat.

from Eq. 1.2. For temperatures above the boiling temperature of $30 \mathrm{~K}$ the measured velocities are in good agreement with this model, but at lower temperatures the clusters are two to three times slower than the prediction of the perfect gas model calculation.

Since the model calculations can describe the cluster velocities very well at high temperatures the model was extended by the use of the van der Waals equation of state instead of the ideal gas law. Assuming an isentropic expansion of the gas the mean fluid velocity was calculated as function of the position in the specific nozzle used in this experiment. Examples for results of these calculations are shown in Fig. 8.

The blue dashed curves represents calculations using the ideal gas equation of state for a perfect gas with constant heat capacity with a stagnation pressure of 10 bar. The lower curve correspond to a stagnation temperature at the inlet of the nozzle of $25 \mathrm{~K}$ whereas the upper curve displays the situation for a temperature of $50 \mathrm{~K}$. Both curves saturate at about one millimetre behind the throat of the nozzle and reaches the value $v_{\max }$ specified by Eq. 1.2. The mass of clusters which are formed by the condensation of the gas molecules will be at some point so large that further scatter processes with gas molecules do not change the cluster velocity any more. At distances larger than this freeze-out position the cluster velocity will therefore be the mean velocity of the gas at this specific position. Since this position will be a few millimetres behind the nozzle throat the measured mean cluster velocities agree well with the maximum velocity of the perfect gas, if the hydrogen temperature at the inlet of the nozzle is higher than the pressure dependent boiling temperature.

The red solid lines show the calculations based on the van der Waals equation of state. It is obvious that the velocity does not saturate any more but increase up to the outlet of the nozzle. 


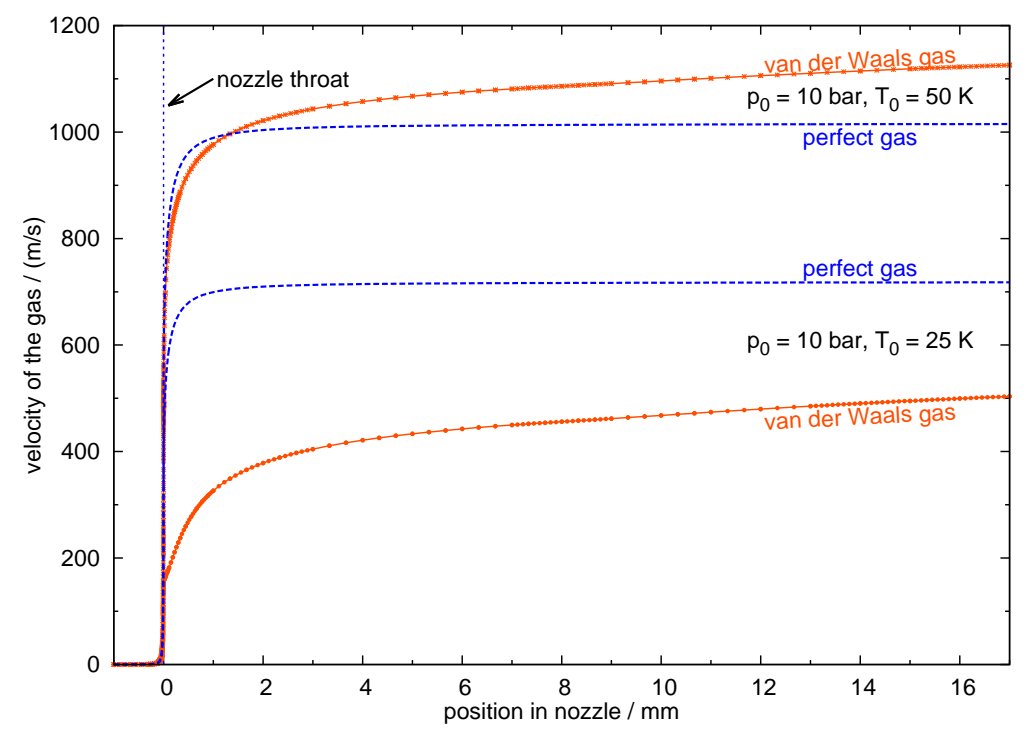

Figure 8: Calculations of the mean fluid velocity assuming different equations of state. The blue dashed lines are calculated based on the ideal gas equation of state with constant heat capacity whereas the red solid lines are calculated using the van der Waals equation of state and the temperature dependent heat capacity of normal hydrogen gas.

Therefore a strong dependence between the location of the freeze-out point and the cluster velocity is expected. In Fig. 7 the velocity is plotted for three different freeze-out positions. The fine solid line shows the calculated velocity at a position of $0.5 \mathrm{~mm}$ from the nozzle throat, the dashed line at one and the dotted line at two millimetres. The measured data taken below the boiling temperature is in very good agreement with the calculated data with a freeze-out position of $0.5 \mathrm{~mm}$ downstream the throat, whereas the data at higher temperatures can be described best with a freeze-out location of two millimetres.

Fig. 9 shows the measured data at an isobar of 17 bar. This pressure is above the pressure at the critical point of 12.964 bar [5] so that the phase change between supercritical fluid and liquid is continuous. The model calculations using the van der Waals model are in very good agreement with the measured data. In this case the lower temperature data below the critical temperature of $33.145 \mathrm{~K}$ [5] can be best described with a freeze-out location of $0.5 \mathrm{~mm}$ and the higher temperature data with a position of about one millimetre behind the nozzle throat.

\section{Summary and outlook}

With the presented time-of-flight technique a very precise method was established to measure the velocity distribution of the clusters produced in a cluster-jet target. Together with the gas dynamic simulations this led to a much better understanding of the cluster production process.

From the comparison between the experimental data and the simulated fluid velocities it is obvious that the cluster production process can be categorized in two regimes. For the operation conditions of conventional cluster-jet targets with relatively high gas temperatures and low pressures at the inlet of the nozzle the hydrogen is gaseous at this point and the clusters are formed 


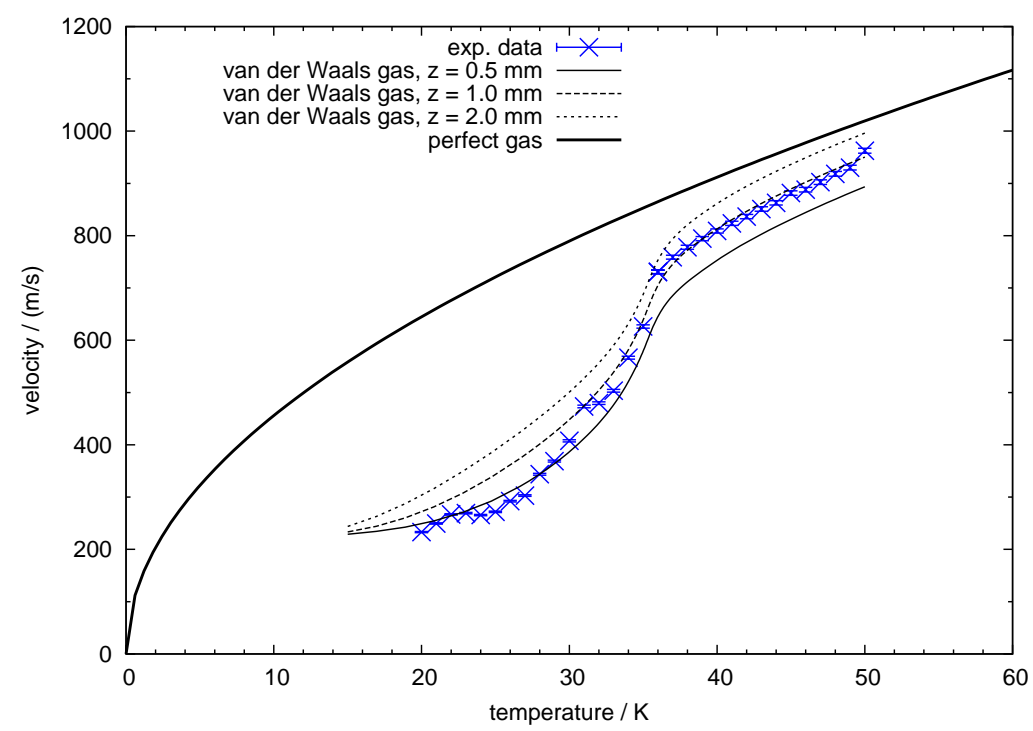

Figure 9: Mean velocity of hydrogen clusters produced in the Münster cluster jet target as function of the hydrogen temperature at the nozzle inlet at a pressure of $17 \mathrm{bar}$.

by condensation. Since this condensation process is a process where several thousand scattering events have to take place in order to accumulate some thousand hydrogen atoms to a single cluster, the freezeout position is some millimetres downwards the nozzle throat. Since this cluster production starts from the gaseous phase, the resulting cluster velocities can be described both with the perfect gas and the van der Waals equation of state. In case of the operation mode accessible with the Münster type cluster-jet target resulting in highest target thickness the hydrogen is liquid at the nozzle inlet. The isentropic expansion process lead to an evaporation of the liquid. In this coexistence region between the liquid and the vapour phase the clusters or nanoparticles are generated as a spray of droplets with random size distribution. The resulting mean cluster velocity are in excellent agreement with simulations based on the van der Waals equation of state.

In future the used time-of-flight method will be extended by the use of an electrostatic spectrometer. This should provide the possibility to measure not only the velocity, but also the mass distribution of the produced clusters. This will be a further important step towards a better understanding of the cluster properties and their underlying production process.

\section{References}

[1] A. Täschner et al., Nucl. Instr. and Meth. A 660 (2011) 22-30, doi:10.1016/j.nima.2011.09.024.

[2] E. Köhler, Design and Performance of the Future Cluster-Jet Target for PANDA at FAIR, in proceedings of STORI'll conference, POS (STORII1) 063

[3] A. Khoukaz, Internal targets for the PANDA Experiment, in proceedings of STORI'11 conference, POS (STORI11) 036

[4] D. Allspach et al., Nucl. Instr. and Meth. A 410 (1998) 195-205.

[5] J. W. Leachman, et al., J. Phys. Chem. Ref. Data 38 (3) (2009) 721-748. 